\title{
On the first Robin eigenvalue of a class of anisotropic operators
}

\author{
Nunzia Gavitone*, Leonardo Trani ${ }^{\dagger}$
}

October 18, 2018

\begin{abstract}
The paper is devoted to the study of some properties of the first eigenvalue of the anisotropic $p$-Laplace operator with Robin boundary condition involving a function $\beta$ which in general is not constant. In particular we obtain sharp lower bounds in terms of the measure of the domain and we prove a monotonicity property of the eigenvalue with respect the set inclusion.
\end{abstract}

KEYWORDS: Eigenvalue problems, nonlinear elliptic equations, Faber-Krahn inequality, Wulff shape, Robin boundary condition

Mathematics Subject Classifications (2010): 35P15, 35P30, 35J60

\section{Introduction}

Let $F$ be a norm in $\mathbb{R}^{n}$, that is a convex, even, 1-homogeneous and non negative function defined in $\mathbb{R}^{n}$. Moreover we will assume that $F \in C^{2}\left(\mathbb{R}^{n} \backslash\{0\}\right)$, and strongly convex that is for $1<p<+\infty$, it holds

$\left[F^{p}\right]_{\xi \xi}(\xi)$ is positive definite in $\mathbb{R}^{n} \backslash\{0\}$.

For $1<p<+\infty$ the so-called anisotropic $p$-Laplacian is defined as follows

$$
\mathcal{Q}_{\mathrm{p}} u:=\operatorname{div}\left(\frac{1}{p} \nabla_{\xi}\left[F^{p}\right](\nabla u)\right) .
$$

The assumptions on $F$ ensure that the operator $\mathcal{Q}_{\mathrm{p}}$ is elliptic. The paper concerns the study of the following Robin eigenvalue problem for $\mathcal{Q}_{\mathrm{p}}$

$$
\begin{cases}-\mathcal{Q}_{\mathrm{p}} v=\ell_{1}(\beta, \Omega)|v|^{p-2} v & \text { in } \Omega \\ F^{p-1}(\nabla v) F_{\xi}(\nabla v) \cdot \nu+\beta(x) F(\nu)|v|^{p-2} v=0 & \text { on } \partial \Omega,\end{cases}
$$

where $\Omega \subset \mathbb{R}^{n}$ is a bounded open set with $C^{1, \alpha}$ boundary, $\left.\alpha \in\right] 0,1[, \nu$ is the Euclidean unit outer normal to $\partial \Omega$ and the function $\beta: \partial \Omega \rightarrow\left[0,+\infty\left[\right.\right.$ belongs to $L^{1}(\partial \Omega)$ and verifies

$$
\int_{\partial \Omega} \beta(x) F(\nu) d \mathcal{H}^{n-1}=m>0 .
$$

\footnotetext{
*Università degli studi di Napoli Federico II, Dipartimento di Matematica e Applicazioni "R. Caccioppoli", Via Cintia, Monte S. Angelo - 80126 Napoli, Italia. Email: nunzia.gavitone@unina.it

${ }^{\dagger}$ Università degli Studi di Napoli Federico II, Dipartimento di Matematica e Applicazioni "R. Caccioppoli", Via Cintia, Monte S. Angelo - 80126 Napoli, Italia. Email: leonardo.trani@unina.it
} 
Here $\ell_{1}(\beta, \Omega)$ is the first Robin eigenvalue of $\mathcal{Q}_{\mathrm{p}}$ and it has the following variational characterization

$$
\ell_{1}(\beta, \Omega)=\inf _{\substack{v \in W^{1, p}(\Omega) \\ v \neq 0}} \frac{\int_{\Omega} F^{p}(\nabla v) d x+\int_{\partial \Omega} \beta(x)|v|^{p} F(\nu) d \mathcal{H}^{n-1}}{\int_{\Omega}|v|^{p} d x},
$$

and the minimizers of (1.2) are weak solutions to the problem (1.1) (see section 3 for the precise definition). When $F(\xi)=|\xi|$ is the Euclidean norm, this problem has been studied for instance in [9, 5, 8, 22, 13, 20]. In particular in [5] and [8] when $\beta(x)=\bar{\beta}$ is a nonnegative constant and for any $p, 1<p<\infty$, the authors prove a sharp lower bound for $\ell_{1}(\bar{\beta}, \Omega)$, keeping fixed the measure of the domain $\Omega$. More precisely, they prove the following Faber-Krahn type inequality

$$
\ell_{1}(\bar{\beta}, \Omega) \geq \ell_{1}\left(\bar{\beta}, B_{R}\right),
$$

where $B_{R}$ is a ball having the same measure than $\Omega$. To prove this result they need mainly two key properties of $\ell_{1}(\bar{\beta}, \Omega)$, that is a level set representation formula and the decreasing monotonicity of $\ell_{1}(\bar{\beta}, \Omega)$ with respect to the radius when $\Omega$ is a ball that is

$$
\ell_{1}\left(\bar{\beta}, B_{r}\right) \leq \ell_{1}\left(\bar{\beta}, B_{s}\right), \quad r>s>0 .
$$

Despite to the Dirichlet eigenvalue, in general $\ell_{1}(\bar{\beta}, \Omega)$ is not monotone decreasing with respect the set inclusion. For instance, in [20] when $p=2$ and $\beta=\bar{\beta}$, the authors prove a sort of monotonicity property (1.4) for suitable convex domains which are not necessary balls and they prove that

$$
\ell_{1}\left(\bar{\beta}, \Omega_{2}\right) \leq \ell_{1}\left(\bar{\beta}, \Omega_{1}\right)
$$

where $\Omega_{1}, \Omega_{2} \subset \mathbb{R}^{n}$ are bounded, Lipschitz and convex domains such that $\Omega_{1} \subset B_{r} \subset \Omega_{2}$. Our aim is to prove (1.3) and (1.5) in the anisotropic case for any $p, 1<p<\infty$ and for a suitable function $\beta$ which is in general, not necessary constant. In particular, regarding (1.3), we will prove the following anisotropic Faber-Krahn inequality

$$
\ell_{1}(\beta, \Omega) \geq \ell_{1}\left(\beta, \mathcal{W}_{R}\right)
$$

where $\mathcal{W}_{R}=\left\{F^{o}(\xi)<R\right\}$, with $F^{o}$ polar norm of $F$, such that $\left|\mathcal{W}_{R}\right|=|\Omega|$ and the function $\beta(x)=w\left(F^{o}(x)\right)$ with $w$ non negative continuous function in $\mathbb{R}$ such that

$$
w(t) \geq C(R) t,
$$

where $C$ is a suitable constant. To do this we need to establish a representation formula for $\ell_{1}(\beta, \Omega)$, for not constant $\beta$. As a consequence of this formula, we also obtain the following anisotropic weighted Cheeger inequality for $\ell_{1}(\beta, \Omega)$

$$
\ell_{1}(\beta, \Omega) \geq h_{\beta}(\Omega)-(p-1)\left\|\beta_{\Omega}^{p^{\prime}}\right\|_{L^{\infty}(\bar{\Omega})},
$$

where $p^{\prime}=\frac{p}{p-1}, \beta_{\Omega}$ is a function defined in the whole $\Omega$ having trace on $\partial \Omega$ equals to $\beta$ and $h_{\beta}(\Omega)$ is the anisotropic weighted Cheeger constant defined in section 6 . This result was proved in the Euclidean case in 22] for $p=2$ and $\beta=\bar{\beta}$ constant.

Our paper has the following structure.

In section 2 we recall notation and preliminary results. In section 3 we prove some basic properties of $\ell_{1}(\beta, \Omega)$. In section 4 we prove some useful properties of the anisotropic radial problem. In section 5 we state and show the quoted monotonicity result for $\ell_{1}(\beta, \Omega)$ and finally in section 6 we prove the representation formula for level set in the general case of variable coefficients $\beta$ proving as applications the quoted Faber-Krahn inequality (1.6) and the anisotropic weighted Cheeger inequality (1.8). 


\section{Notation and preliminaries}

\section{$2.1 \quad$ Finsler norm}

Let $F$ be a convex, even, 1-homogeneous and non negative function defined in $\mathbb{R}^{n}$. Then $F$ is a convex function such that

$$
F(t \xi)=|t| F(\xi), \quad t \in \mathbb{R}, \xi \in \mathbb{R}^{n},
$$

and such that

$$
a|\xi| \leq F(\xi), \quad \xi \in \mathbb{R}^{n},
$$

for some constant $a>0$. The hypotheses on $F$ imply there exists $b \geq a$ such that

$$
F(\xi) \leq b|\xi|, \quad \xi \in \mathbb{R}^{n} .
$$

Moreover, throughout the paper we will assume that $F \in C^{2}\left(\mathbb{R}^{n} \backslash\{0\}\right)$, and

$$
\left[F^{p}\right]_{\xi \xi}(\xi) \text { is positive definite in } \mathbb{R}^{n} \backslash\{0\},
$$

with $1<p<+\infty$.

The polar function $F^{o}: \mathbb{R}^{n} \rightarrow[0,+\infty[$ of $F$ is defined as

$$
F^{o}(v)=\sup _{\xi \neq 0} \frac{\langle\xi, v\rangle}{F(\xi)} .
$$

It is easy to verify that also $F^{o}$ is a convex function which satisfies properties (2.1) and (2.2) . Furthermore,

$$
F(v)=\sup _{\xi \neq 0} \frac{\langle\xi, v\rangle}{F^{o}(\xi)} .
$$

The above property implies the following anisotropic version of the Cauchy Schwartz inequality

$$
|\langle\xi, \eta\rangle| \leq F(\xi) F^{o}(\eta), \quad \forall \xi, \eta \in \mathbb{R}^{n} .
$$

The set

$$
\mathcal{W}=\left\{\xi \in \mathbb{R}^{n}: F^{o}(\xi)<1\right\}
$$

is the so-called Wulff shape centered at the origin. We put $\kappa_{n}=|\mathcal{W}|$, where $|\mathcal{W}|$ denotes the Lebesgue measure of $\mathcal{W}$. More generally, we denote by $\mathcal{W}_{r}\left(x_{0}\right)$ the set $r \mathcal{W}+x_{0}$, that is the Wulff shape centered at $x_{0}$ with measure $\kappa_{n} r^{n}$, and $\mathcal{W}_{r}(0)=\mathcal{W}_{r}$.

The following properties of $F$ and $F^{o}$ hold true:

$$
\begin{aligned}
& \left\langle F_{\xi}(\xi), \xi\right\rangle=F(\xi), \quad\left\langle F_{\xi}^{o}(\xi), \xi\right\rangle=F^{o}(\xi), \quad \forall \xi \in \mathbb{R}^{n} \backslash\{0\}, \\
& F\left(F_{\xi}^{o}(\xi)\right)=F^{o}\left(F_{\xi}(\xi)\right)=1, \quad \forall \xi \in \mathbb{R}^{n} \backslash\{0\}, \\
& F^{o}(\xi) F_{\xi}\left(F_{\xi}^{o}(\xi)\right)=F(\xi) F_{\xi}^{o}\left(F_{\xi}(\xi)\right)=\xi \quad \forall \xi \in \mathbb{R}^{n} \backslash\{0\} .
\end{aligned}
$$

\section{$2.2 \quad$ Anisotropic perimeter}

We recall the definition of anisotropic perimeter for a bounded, Lipschitz open set:

Definition 2.1. Let $K$ be a bounded open subset of $\mathbb{R}^{n}$ with Lipschitz boundary. The anisotropic perimeter of $K$ is:

$$
P_{F}(K)=\int_{\partial K} F(\nu) d \mathcal{H}^{n-1}
$$

where $\nu$ denotes the unit outer normal to $\partial K$ and $\mathcal{H}^{n-1}$ is the $(n-1)$-dimensional Hausforff measure. 
Clearly, the perimeter of $K$ is finite if and only if the usual Euclidean perimeter of $K, P_{\mathcal{E}}(K)$ is finite. Indeed, by the quoted properties of $F$ we obtain that

$$
a P_{\mathcal{E}}(K) \leq P_{F}(K) \leq b P_{\mathcal{E}}(K) .
$$

Furthermore, an isoperimetric inequality for the anisotropic perimeter holds (see for instance [6, 1, 17]). Namely let $\mathrm{K}$ be a bounded open subset of $\mathbb{R}^{n}$ with Lipschitz boundary, then

$$
P_{F}(K) \geq n \kappa_{n}^{\frac{1}{n}}|K|^{1-\frac{1}{n}}
$$

where $\kappa_{n}$ is the Lebesgue measure of the unit Wulff shape. In particular, the equality in (2.5) holds if and only if the set $K$ is homothetic to a Wulff shape. We recall the following so-called weighted anisotropic isoperimetric inequality (see for instance [3] and [4])

$$
\int_{\partial \Omega} f\left(F^{o}(x)\right) F(\nu) d \mathcal{H}^{n-1} \geq \int_{\partial \mathcal{W}_{R}} f\left(F^{o}(x)\right) F(\nu) d \mathcal{H}^{n-1}=f(R) P_{F}\left(\mathcal{W}_{R}\right),
$$

where $\mathcal{W}_{R}$ is a Wulff shape such that $|\Omega|=\left|\mathcal{W}_{R}\right|$ and $f:[0, R] \rightarrow[0,+\infty[$ is a nondecreasing function such that

$$
g(z)=f\left(z^{\frac{1}{n}}\right) z^{1-\frac{1}{n}}, \quad 0 \leq z \leq R^{n},
$$

is convex with respect to $z$.

If $\Omega \subset \mathbb{R}^{n}$ is a bounded open set, the anisotropic Cheeger constant of $\Omega$ is defined as follows

$$
h_{F}(\Omega)=\inf _{U \subset \Omega} \frac{P_{F}(U)}{|U|} .
$$

In [10] the authors prove that

$$
\frac{1}{R_{F}} \leq h_{F}(\Omega) \leq \frac{n}{R_{F}},
$$

where $R_{F}$ is the anisotropic inradius that is the radius of the biggest Wulff shape contained in $\Omega$.

\subsection{Anisotropic $p$-Laplacian}

Let $\Omega \subset \mathbb{R}^{n}$ be a bounded open set and $u \in W^{1, p}(\Omega)$. For $1<p<+\infty$ the anisotropic $p$ Laplacian is defined as follows

$$
\mathcal{Q}_{\mathrm{p}} u:=\operatorname{div}\left(\frac{1}{p} \nabla_{\xi}\left[F^{p}\right](\nabla u)\right) .
$$

The hypothesis (2.4) on $F$ ensures that the operator is elliptic, hence there exists a positive constant $\gamma$ such that

$$
\frac{1}{p} \sum_{i, j=1}^{n} \nabla_{\xi_{i} \xi_{j}}^{2}\left[F^{p}\right](\eta) \xi_{i} \xi_{j} \geq \gamma|\eta|^{p-2}|\xi|^{2},
$$

for any $\eta \in \mathbb{R}^{n} \backslash\{0\}$ and for any $\xi \in \mathbb{R}^{n}$.

For $p=2, \mathcal{Q}_{2}$ is the so-called Finsler Laplacian, and when $F(\xi)=|\xi|=\sqrt{\sum_{i=1}^{n} x_{i}^{2}}$ is the Euclidean norm, $\mathcal{Q}_{\mathrm{p}}$ reduces to the well known $p$-Laplace operator.

Let $\Omega$ be a bounded open set in $\mathbb{R}^{n}, n \geq 2,1<p<+\infty$, and consider the following eigenvalue problem with Dirichlet boundary conditions related to $\mathcal{Q}_{\mathrm{p}}$

$$
\begin{cases}-\mathcal{Q}_{\mathrm{p}} u=\lambda|u|^{p-2} u & \text { in } \Omega \\ u=0 & \text { on } \partial \Omega .\end{cases}
$$


The smallest eigenvalue, denoted by $\lambda_{D}(\Omega)$, has the following well-known variational characterization:

$$
\lambda_{D}(\Omega)=\min _{\varphi \in W_{0}^{1, p}(\Omega) \backslash\{0\}} \frac{\int_{\Omega} F^{p}(\nabla \varphi) d x}{\int_{\Omega}|\varphi|^{p} d x} .
$$

For the first eigenvalue of the anisotropic $p$-Laplacian with Dirichlet boundary conditions, the following isoperimetric inequality holds (see [2]).

Theorem 2.1. Let $\Omega \subset \mathbb{R}^{n}$, be a bonded domain with $n \geq 2$ then

$$
|\Omega|^{\frac{p}{n}} \lambda_{D}(\Omega) \geq \kappa_{n}^{\frac{p}{n}} \lambda_{D}(\mathcal{W}) .
$$

Moreover, the equality holds if and only if $\Omega$ is homothetic to a Wulff shape.

Finally we recall that for a given bounded open set in $\mathbb{R}^{n}$, the anisotropic Cheeger inequality states that (see for instance [7, 10, 21])

$$
\lambda_{D}(\Omega) \geq\left(\frac{h_{F}(\Omega)}{p}\right)^{p}, \quad 1<p<\infty .
$$

\section{The first Robin eigenvalue of $\mathcal{Q}_{\mathrm{p}}$}

In this section we will investigate some properties of the first Robin eigenvalue related to $\mathcal{Q}_{\mathrm{p}}$, $1<p<\infty$. From now on we assume that

$$
\left.\Omega \subset \mathbb{R}^{n} \text { is a bounded open set with } C^{1, \alpha} \text { boundary and } \alpha \in\right] 0,1[\text {. }
$$

Let us consider the following Robin eigenvalue problem for $\mathcal{Q}_{\mathrm{p}}$

$$
\begin{cases}-\mathcal{Q}_{\mathrm{p}} u=\ell|u|^{p-2} u & \text { in } \Omega \\ F^{p-1}(\nabla u) F_{\xi}(\nabla u) \cdot \nu+\beta(x) F(\nu)|u|^{p-2} u=0 & \text { on } \partial \Omega,\end{cases}
$$

where $u \in W^{1, p}(\Omega), \nu$ is the Euclidean unit outer normal to $\partial \Omega$ and the function $\beta: \partial \Omega \rightarrow$ $\left[0,+\infty\left[\right.\right.$ belongs to $L^{1}(\partial \Omega)$ and verifies

$$
\int_{\partial \Omega} \beta(x) F(\nu) d \mathcal{H}^{n-1}=m>0 .
$$

From now on we will write $\bar{\beta}$ instead of $\beta$ when $\beta$ is a positive constant.

Definition 3.1. A function $u \in W^{1, p}(\Omega), u \not \equiv 0$ is an eigenfunction to (3.2) if $\beta(\cdot)|u|^{p} \in L^{1}(\partial \Omega)$ and

$$
\int_{\Omega} F^{p-1}(\nabla v) F_{\xi}(\nabla v) \cdot \nabla \varphi d x+\int_{\partial \Omega} \beta(x)|u|^{p-2} u \varphi F(\nu) d \mathcal{H}^{n-1}=\ell \int_{\Omega}|u|^{p-2} u \varphi d x
$$

for any test function $\varphi \in W^{1, p}(\Omega) \cap L^{\infty}(\partial \Omega)$. The corresponding number $\ell$, is called Robin eigenvalue.

The smallest eigenvalue of (3.2), $\ell_{1}(\beta, \Omega)$ has the following variational characterization

$$
\ell_{1}(\beta, \Omega)=\inf _{\substack{v \in W^{1, p}(\Omega) \\ v \neq 0}} J[\beta, v]:=\inf _{\substack{v \in W^{1, p}(\Omega) \\ v \neq 0}} \frac{\int_{\Omega} F^{p}(\nabla v) d x+\int_{\partial \Omega} \beta(x)|v|^{p} F(\nu) d \mathcal{H}^{n-1}}{\int_{\Omega}|v|^{p} d x} .
$$


By definition we have

$$
\ell_{1}(\beta, \Omega) \leq \lambda_{D}(\Omega)
$$

where $\lambda_{D}(\Omega)$ is the first Dirichlet eigenvalue of $\mathcal{Q}_{\mathrm{p}}$. Indeed choosing as test function in (3.5), the first Dirichlet eigenfunction $u_{D}$ of $\lambda_{D}(\Omega)$ in the Reileigh quotient, we get

$$
\begin{aligned}
\ell_{1}(\beta, \Omega)= & \min _{\substack{v \in W^{1, p}(\Omega) \\
v \neq 0}} \frac{\int_{\Omega}[F(\nabla v)]^{p} d x+\int_{\partial \Omega} \beta|v|^{p} F(\nu) d \mathcal{H}^{n-1}}{\int_{\Omega}|v|^{p} d x} \\
& \leq \frac{\int_{\Omega}\left[F\left(\nabla u_{D}\right)\right]^{p} d x+\int_{\partial \Omega} \beta\left|u_{D}\right|^{p} F(\nu) d \mathcal{H}^{n-1}}{\int_{\Omega}\left|u_{D}\right|^{p} d x}=\frac{\int_{\Omega}\left[F\left(\nabla u_{D}\right)\right]^{p} d x}{\int_{\Omega}\left|u_{D}\right|^{p} d x}=\lambda_{D}(\Omega) .
\end{aligned}
$$

The following existence result holds.

Proposition 3.1. Let $\beta \in L^{1}(\partial \Omega), \beta \geq 0$ be such that (3.3) holds. Then there exists a positive minimizer $u \in C^{1, \alpha}(\Omega) \cap L^{\infty}(\Omega)$ of (3.5) which is a weak solution to (3.2) in $\Omega$ with $\ell=\ell_{1}(\beta, \Omega)$. Moreover $\ell_{1}(\beta, \Omega)$ is positive and it is simple, that is the relative eigenfunction $u$ is unique up to a multiplicative constant.

Proof. Let $u_{k} \in W^{1, p}(\Omega)$ be a minimizing sequence of (3.5) such that $\left\|u_{k}\right\|_{L^{p}(\Omega)}=1$. Then, being $u_{k}$ bounded in $W^{1, p}(\Omega)$ there exists a subsequence, still denoted by $u_{k}$ and a function $u \in W^{1, p}(\Omega)$ with $\|u\|_{L^{p}(\Omega)}=1$, such that $u_{k} \rightarrow u$ strongly in $L^{p}(\Omega)$ and $\nabla u_{k} \rightarrow \nabla u$ weakly in $L^{p}(\Omega)$. Then $u_{k}$ converges to $u$ in $L^{p}(\partial \Omega)$ and then almost everywhere on $\partial \Omega$ to $u$. Then by the weak lower semicontinuity and Fatou's lemma we get

$$
\ell_{1}(\beta, \Omega)=\lim _{k \rightarrow+\infty} J\left[\beta, u_{k}\right] \geq J[\beta, u],
$$

then $\beta(\cdot)|u|^{p} \in L^{1}(\partial \Omega)$ and $u$ is an eigenfunction related to $\ell_{1}(\beta, \Omega)$ by definition. Moreover $u \in L^{\infty}(\Omega)$. To see that, we can argue exactly as in [11] in order to get that $u \in L^{\infty}(\Omega)$.

Now the $L^{\infty}$-estimate, the hypothesis (2.4) and the properties of $F$ allow to apply standard regularity results (see [16], [27]), in order to obtain that $u \in C^{1, \alpha}(\Omega)$.

In order to prove that $\ell_{1}(\beta, \Omega)>0$, we procede by contraddiction supposing that there exists $\beta_{o}$ which verifies (3.3) and such that $\ell_{1}\left(\beta_{o}, \Omega\right)=0$. Then there exists $u_{\beta_{o}} \in C^{1, \alpha}(\Omega) \cap L^{\infty}(\bar{\Omega})$ such that $u_{\beta_{o}} \geq 0,\left\|u_{\beta_{o}}\right\|_{L^{p}(\Omega)}=1$ and

$$
0=\ell_{1}\left(\beta_{o}, \Omega\right)=\int_{\Omega} F^{p}\left(\nabla u_{\beta_{o}}\right) d x+\int_{\partial \Omega} \beta_{o} u_{\beta_{o}}^{p} F(\nu) d \mathcal{H}^{n-1} .
$$

Then $u_{\beta_{o}}$ has to be constant in $\bar{\Omega}$ and then $u_{\beta_{o}}^{p} \int_{\partial \Omega} \beta_{o} F(\nu)=u_{\beta_{o}}^{p} m=0$. Being $m>0$, then $u_{\beta_{o}}=0$ in $\bar{\Omega}$, and this is not true. Hence $\ell_{1}\left(\beta_{o}, \Omega\right)>0$.

Finally to prove the semplicity of the eigenfunctions we can procede exactly as in [11]. For completeness we recall the main steps. Let $u, w$ be positive minimizers of the functional $J$ defined in 3.5 such that $\|u\|_{p}=\|w\|_{p}=1$, and let us consider the function $\eta_{t}=\left(t u^{p}+(1-t) w^{p}\right)^{1 / p}$, with $t \in[0,1]$. Obviously, $\left\|\eta_{t}\right\|_{p}=1$. Clearly it holds:

$$
J[\beta, u]=\ell_{1}(\beta, \Omega)=J[\beta, w] .
$$

In order to compute $J\left[\beta, \eta_{t}\right]$ we observe that by using the homogeneity and the convexity of $F$ it is not hard to prove that (see for instance [11] for the precise computation)

$$
F^{p}\left(\nabla \eta_{t}\right) \leq t F^{p}(\nabla v)+(1-t) F^{p}(\nabla w) .
$$


Hence recalling (3.6), we obtain

$$
J\left[\beta, \eta_{t}\right] \leq t J[\beta, u]+(1-t) J[\beta, w]=\ell_{1}(\beta, \Omega),
$$

and then $\eta_{t}$ is a minimizer for $J$. This implies that the equality holds in (3.7), and as showed in [11], this implies that $u=w$ that is the uniqueness.

The following result characterizes the first eigenfunctions.

Proposition 3.2. Let $\beta \in L^{1}(\partial \Omega), \beta \geq 0$ be such that (3.3) holds. Let $\eta>0$ and $v \in W^{1, p}(\Omega)$, $v \not \equiv 0$ and $v \geq 0$ in $\Omega$ such that

$$
\begin{cases}-\mathcal{Q}_{\mathrm{p}} v=\eta v^{p-1} & \text { in } \Omega \\ F^{p-1}(\nabla v) F_{\xi}(D v) \cdot \nu+\beta F(\nu) v^{p-1}=0 & \text { on } \partial \Omega\end{cases}
$$

in the sense of Definition 3.1. Then $v$ is a first eigenfunction of (6.1), and $\eta=\ell_{1}(\beta, \Omega)$.

Proof. Let $u \in W^{1, p}(\Omega)$ be a positive eigenfunction related to $\ell_{1}(\beta, \Omega)$. Choosing $u^{p} /(v+\varepsilon)^{p-1}$, with $\varepsilon>0$, as test function in the Definition 3.1 for the solution $v$, and arguing exactly as in [11, we get the claim.

Remark 3.1. We observe that Propositions 3.1 and 3.2 generalize the results proved respectively in [13] for the Euclidean norm and in [11] when $\beta(x)=\beta$ is a positive constant.

Theorem 3.1. Let $\beta \in L^{1}(\partial \Omega), \beta \geq 0$ and such that (3.3) holds. The following properties hold for $\ell_{1}(\beta, \Omega)$

(i) $\forall t>0, \ell_{1}\left(\beta\left(\frac{x}{t}\right), t \Omega\right)=t^{-p} \ell_{1}\left(t^{p-1} \beta(y), \Omega\right), \quad x \in \partial(t \Omega), y \in \partial \Omega$;

(ii) $\ell_{1}(\beta, \Omega) \leq \frac{m}{|\Omega|}$

(iii) $a^{p} \ell_{\mathcal{E}}\left(a^{1-p} \beta, \Omega\right) \leq \ell_{1}(\beta, \Omega) \leq b^{p} \ell_{\mathcal{E}}\left(b^{1-p} \beta, \Omega\right)$,

where $a, b$ are defined in (2.2), (2.3) and $\ell_{\mathcal{E}}\left(a^{1-p} \beta, \Omega\right), \ell_{\mathcal{E}}\left(b^{1-p} \beta, \Omega\right)$ are the first Robin eigenvalue for the Euclidean p-Laplacian corresponding respectively to the function $a^{1-p} \beta$ and $b^{1-p} \beta$;

(iv) If $\beta(x) \geq \bar{\beta}>0$, for almost $x \in \partial \Omega$, then

$$
\sup _{|\Omega|=k} \ell_{1}(\beta, \Omega)=+\infty
$$

Proof. By the homogeneity of $F$, we have:

$$
\begin{aligned}
& \ell_{1}\left(\beta\left(\frac{x}{t}\right), t \Omega\right)=\min _{\substack{\varphi \in W^{1, p}(t \Omega) \\
\varphi \neq 0}} \frac{\int_{t \Omega} F^{p}(\nabla \varphi(x)) d x+\int_{\partial(t \Omega)} \beta\left(\frac{x}{t}\right)|\varphi(x)|^{p} F(\nu(x)) d \mathcal{H}^{n-1}(x)}{\int_{t \Omega}|\varphi(x)|^{p} d x} \\
& =\min _{\substack{v \in W^{1, p}(\Omega) \\
v \neq 0}} \frac{t^{-p} \int_{t \Omega} F^{p}\left(\nabla v\left(\frac{x}{t}\right)\right) d y+\left.\int_{\partial(t \Omega)} \beta\left(\frac{x}{t}\right)\left|v\left(\frac{x}{t}\right)\right|\right|^{p} F\left(\nu\left(\frac{x}{t}\right)\right) d \mathcal{H}^{n-1}(y)}{t^{n} \int_{\Omega}|v(y)|^{p} d y}= \\
& =\min _{\substack{v \in W^{1, p}(\Omega) \\
v \neq 0}} \frac{t^{n-p} \int_{\Omega} F^{p}(\nabla v(y)) d y+t^{n-1} \int_{\partial \Omega} \beta(y)|v(y)|^{p} F(\nu(y)) d \mathcal{H}^{n-1}(y)}{t^{n} \int_{\Omega}|v(y)|^{p} d y}=t^{-p} \ell_{1}\left(t^{p-1} \beta(y), \Omega\right) .
\end{aligned}
$$


In order to obtain the second property, it is sufficient to consider a non-zero constant as test function in (3.5).

Now we prove the inequality in the right-hand side in (iii). The proof of the other inequality is similar. By using (3.5) and (2.3), we obtaine that

$$
\begin{aligned}
& \ell_{1}(\beta, \Omega)= \inf _{\substack{v \in W^{1, p}(\Omega) \\
v \neq 0}} \frac{\int_{\Omega} F^{p}(\nabla v) d x+\int_{\partial \Omega} \beta(x)|v|^{p} F(\nu) d \mathcal{H}^{n-1}}{\int_{\Omega}|v|^{p} d x} \leq \\
& \inf _{\substack{v \in W^{1, p}(\Omega) \\
v \neq 0}} b^{p} \frac{\int_{\Omega}|\nabla v|^{p} d x+b^{1-p} \int_{\partial \Omega} \beta(x)|v|^{p} d \mathcal{H}^{n-1}}{\int_{\Omega}|v|^{p} d x}= \\
& b^{p} \inf _{\substack{v \in W^{1, p}(\Omega) \\
v \neq 0}} \frac{\int_{\Omega}|\nabla v|^{p} d x+\int_{\partial \Omega} b^{1-p} \beta(x)|v|^{p} d \mathcal{H}^{n-1}}{\int_{\Omega}|v|^{p} d x}=b^{p} \ell_{\mathcal{E}}\left(b^{1-p} \beta, \Omega\right),
\end{aligned}
$$

where last equality follows, by definition of $\ell_{\mathcal{E}}\left(b^{1-p} \beta, \Omega\right)$.

Finally we give the proof of $(i v)$. Clearly $\ell_{1}(\beta, \Omega) \geq \ell_{1}(\bar{\beta}, \Omega)$, then by [11, Proposition 3.1], we know that

$$
\ell_{1}(\bar{\beta}, \Omega) \geq\left(\frac{p-1}{p}\right)^{p} \frac{\bar{\beta}}{R_{F}\left(1+\bar{\beta}^{\frac{1}{p-1}} R_{F}\right)}
$$

where $R_{F}$ is the anisotropic inradius of the subset $\Omega$. The claim follows constructing a sequence of convex sets $\Omega_{k}$ with $\left|\Omega_{k}\right|=1$ and such that $R_{F}\left(\Omega_{k}\right) \rightarrow 0$, for $k \rightarrow \infty$. Let $k>0$, proceeding as in [10, 12], it is possible to consider the $n$-rectangles $\left.\Omega_{k}=\right]-\frac{1}{2 k}, \frac{1}{2 k}[\times]-\frac{k^{\frac{1}{n-1}}}{2}, \frac{k^{\frac{1}{n-1}}}{2}\left[{ }^{n-1}\right.$ and suppose that $R_{F}\left(\Omega_{k}\right)=\frac{1}{2 k} F^{o}\left(e_{1}\right)$. Then we obtain

$$
\ell_{1}\left(\bar{\beta}, \Omega_{k}\right) \geq\left(\frac{p-1}{p}\right)^{p} \frac{4 k^{2} \bar{\beta}}{F^{o}\left(e_{1}\right)\left(2 k+\bar{\beta}^{\frac{1}{p-1}} F^{o}\left(e_{1}\right)\right)} \rightarrow+\infty \text { for } k \rightarrow \infty .
$$

\section{The anisotropic radial case}

In this section we recall some properties of the first eigenvalue of $\mathcal{Q}_{\mathrm{p}}$ with Robin boundary condition when $\Omega$ is a Wulff shape. We suppose that $\beta=\bar{\beta}$ is a positive constant then we consider

$$
\ell_{1}\left(\bar{\beta}, \mathcal{W}_{R}\right)=\min _{\substack{v \in W^{1, p}\left(\mathcal{W}_{R}\right) \\ v \neq \equiv 0}} J(\bar{\beta}, v)=\min _{\substack{v \in W^{1, p}\left(\mathcal{W}_{R}\right) \\ v \neq 0}} \frac{\int_{\mathcal{W}_{R}}[F(\nabla v)]^{p} d x+\bar{\beta} \int_{\partial \mathcal{W}_{R}}|v|^{p} F(\nu) d \mathcal{H}^{n-1}}{\int_{\mathcal{W}_{R}}|v|^{p} d x},
$$

where $\mathcal{W}_{R}=R \mathcal{W}=\left\{x: F^{o}(x)<R\right\}$, with $R>0$, and $\mathcal{W}$ is the Wulff shape centered at the origin.

By Proposition 3.1, the minimizers of (4.1) solve the following problem:

$$
\begin{cases}-\mathcal{Q}_{\mathrm{p}} v=\ell_{1}\left(\bar{\beta}, \mathcal{W}_{R}\right)|v|^{p-2} v & \text { in } \mathcal{W}_{R}, \\ F^{p-1}(\nabla v) F_{\xi}(D v) \cdot \nu+\bar{\beta} F(\nu)|v|^{p-2} v=0 & \text { on } \partial \mathcal{W}_{R} .\end{cases}
$$

In [11, 9, 5] the authors prove the following result 
Theorem 4.1. Let $v_{p} \in C^{1, \alpha}\left(\mathcal{W}_{R}\right) \cap C\left(\overline{\mathcal{W}_{R}}\right)$ be a positive solution to problem (4.2). Then $v_{p}(x)=\varrho_{p}\left(F^{o}(x)\right)$, with $x \in \overline{\mathcal{W}}_{R}$, where $\varrho_{p}(r), r \in[0, R]$, is a decreasing function such that $\varrho_{p} \in C^{\infty}(0, R) \cap C^{1}([0, R])$ and it verifies

$$
\left\{\begin{array}{l}
\left.-(p-1)\left(-\varrho_{p}^{\prime}(r)\right)^{p-2} \varrho_{p}^{\prime \prime}(r)+\frac{n-1}{r}\left(-\varrho_{p}^{\prime}(r)\right)^{p-1}=\ell_{1}\left(\bar{\beta}, \mathcal{W}_{R}\right) \varrho_{p}(r)^{p-1}, \quad r \in\right] 0, R[, \\
\varrho_{p}^{\prime}(0)=0, \\
-\left(-\varrho_{p}^{\prime}(R)\right)^{p-1}+\bar{\beta}\left(\varrho_{p}(R)\right)^{p-1}=0 .
\end{array}\right.
$$

Remark 4.1. We observe that the first eigenvalue in the Wulff $\mathcal{W}_{R}=\left\{F^{o}(x)<R\right\}$ is the same for any norm $F$. In particular it coincides with the first Robin eigenvalue in the Euclidean ball $B_{R}$ for the $p$-Laplace operator. Finally we emphasize that in this case the eigenfunctions have more regularity because $\bar{\beta}$ is a positive constant.

Theorem 4.1, as in [5, 11, suggests to consider, for every $x \in \mathcal{W}_{R}$, the following function

$$
f\left(r_{x}\right)=\frac{\left(-\rho_{p}^{\prime}\left(r_{x}\right)\right)^{p-1}}{\left(\rho_{p}\left(r_{x}\right)\right)^{p-1}}=\frac{\left[F\left(\nabla v_{p}(x)\right)\right]^{p-1}}{v_{p}(x)^{p-1}}=\frac{\left[F\left(\nabla v_{p}(x)\right)\right]^{p-1} F_{\xi}\left(D v_{p}(x)\right) \cdot \nu}{v_{p}(x)^{p-1} F(\nu)},
$$

where

$$
r_{x}=F^{o}(x), \quad 0 \leq r_{x} \leq R .
$$

Let us observe that $f$ is nonnegative, $f(0)=0$ and

$$
f(R)=\frac{\left(-\rho_{p}^{\prime}(R)\right)^{p-1}}{\left(\rho_{p}(R)\right)^{p-1}}=\frac{\left[F\left(\nabla v_{p}(x)\right)\right]^{p-1}}{v_{p}(x)^{p-1}}=\frac{\left[F\left(\nabla v_{p}(x)\right)\right]^{p-1} F_{\xi}\left(\nabla v_{p}(x)\right) \cdot \nu}{v_{p}(x)^{p-1} F(\nu)}=\bar{\beta}
$$

The following result proved in the Euclidean case in [5] and in [11] in the anisotropic case, states that the first Robin eigenvalue is monotone decreasing with respect the set inclusion in the class of Wulff shapes.

Lemma 4.1. The function $r \rightarrow \ell_{1}\left(\bar{\beta}, \mathcal{W}_{r}\right)$ is strictly decreasing in $] 0, \infty[$.

In [5] and [11] the authors prove also the following monotonicity property for the function $f$ defined in (4.4).

Lemma 4.2. Let $f$ be the function defined in (4.4). Then $f(r)$ is strictly increasing in $[0, R]$.

In the next result we prove a convex property for the function $f$.

Theorem 4.2. Let $f$ be the function defined in (4.4). Then the function

$$
g(z)=f\left(z^{\frac{1}{n}}\right) z^{1-\frac{1}{n}}, \quad 0 \leq z \leq R^{n},
$$

is convex with respect to $z$.

Proof. We first observe that by (4.3) it holds

$$
\begin{aligned}
f^{\prime}(r)=\frac{d}{d r}\left(\frac{-\rho_{p}^{\prime}(r)}{\rho_{p}(r)}\right)^{p-1}=(p & -1) f^{\frac{p-2}{p-1}}\left(\frac{-\rho_{p}^{\prime \prime}}{\rho_{p}}+\left(\frac{\rho_{p}^{\prime}}{\rho_{p}}\right)^{2}\right) \\
& \left.=\ell_{1}\left(\bar{\beta}, \mathcal{W}_{R}\right)-\frac{(n-1)}{r} f+(p-1) f^{\frac{p}{p-1}} \quad \forall r \in\right] 0, R[.
\end{aligned}
$$


Then

$$
\begin{aligned}
g^{\prime}(z)=\frac{1}{n} f^{\prime}\left(z^{\frac{1}{n}}\right)+\frac{(n-1)}{n} \frac{f\left(z^{\frac{1}{n}}\right)}{z^{\frac{1}{n}}} & \\
=\frac{1}{n}\left(\ell_{1}\left(\bar{\beta}, \mathcal{W}_{R}\right)-\frac{(n-1)}{z^{\frac{1}{n}}} f\left(z^{\frac{1}{n}}\right)+(p-1) f^{\frac{p}{p-1}}\left(z^{\frac{1}{n}}\right)\right) & +\frac{(n-1)}{n} \frac{f\left(z^{\frac{1}{n}}\right)}{z^{\frac{1}{n}}} \\
& =\frac{\ell_{1}\left(\bar{\beta}, \mathcal{W}_{R}\right)}{n}+\frac{(p-1)}{n} f^{\frac{p}{p-1}}\left(z^{\frac{1}{n}}\right),
\end{aligned}
$$

which is increasing and this implies the thesis.

Finally the following comparison result for $f$ holds

Theorem 4.3. Let $f$ be the function defined in (4.4). Then there exists a positive constant $C=C(R)$ such that

$$
f(r) \leq r C(R), \text { for } 0 \leq r \leq R
$$

Proof. By (4.5) and by Lemma 4.2 we obtain that $f$ verifies the following equation

$$
f^{\prime}(r)=\ell_{1}\left(\bar{\beta}, \mathcal{W}_{R}\right)-\frac{(n-1)}{r} f(r)+(p-1) f^{\frac{p}{p-1}}(r) \leq C(R)-\frac{(n-1)}{r} f(r)
$$

where

$$
C(R)=\ell_{1}\left(\bar{\beta}, \mathcal{W}_{R}\right)+(p-1) f(R)^{\frac{p}{p-1}}=\ell_{1}\left(\bar{\beta}, \mathcal{W}_{R}\right)+(p-1) \bar{\beta}^{\frac{p}{p-1}}
$$

Then by (4.6) multiplying both sides by $r^{n-1}$ we get

$$
f^{\prime}(r) r^{n-1}+(n-1) r^{n-2} f(r) \leq C(R) r^{n-1},
$$

and

$$
\frac{d}{d r}\left(r^{n-1} f(r)\right) \leq C(R) r^{n-1}
$$

Then the claim follows integrating both sides between 0 and $r$.

Remark 4.2. The results contained in Lemma 4.2 and Theorem 4.2 ensures that $f(r)$ is an admissible weight for the weighted anisotropic isoperimetric inequality quoted in (2.6)

\section{$5 \quad$ A monotonicity property for $\ell_{1}(\bar{\beta} ; \Omega)$}

In this section we assume that $\beta=\bar{\beta}$ is a positive constant. The first Robin eigenvalue $\ell_{1}(\bar{\beta}, \Omega)$ has not, in general, a monotonicity property with respect the set inclusion. For instance in [15] in the Euclidean case, for the Laplace operator, the authors give a counterexample. More precisely, they construct a suitable sequence of sets $\Omega_{k}$ such that $P_{\mathcal{E}}\left(\Omega_{k}\right) \rightarrow \infty, B_{1}(0) \subset \Omega_{k} \subset B_{1+\varepsilon}(0)$ which verify

$$
\ell_{\mathcal{E}}\left(\bar{\beta}, \Omega_{k}\right)>\ell_{\mathcal{E}}\left(\bar{\beta}, B_{1}(0)\right)>\ell_{\mathcal{E}}\left(\bar{\beta}, B_{2}(0)\right) .
$$

Here $B_{r}\left(x_{o}\right)$ denotes the Euclidean ball with radius $r$ and centered at the pint $x_{o}$ and $\lambda_{\mathcal{E}}\left(B_{1+\varepsilon}(0)\right)$ is the Euclidean first Dirichlet eigenvalue of the Laplacian of the ball $B_{1+\varepsilon}(0)$. In what follows we prove a monotonicity type property for the first Robin eigenvalue of the operator $\mathcal{Q}_{\mathrm{p}}$ with respect the set inclusion. In the Euclidean case for the Laplace operator we refer the reader for instance to [20]. 
Theorem 5.1. Let $\Omega \subset \mathbb{R}^{n}$ be a bounded open set with $C^{1, \alpha}$ boundary, $\left.\alpha \in\right] 0,1\left[\right.$. Let $\mathcal{W}_{R}$ be a Wulff shape such that $\Omega \subset \mathcal{W}_{R}$ and $\bar{\beta}$ a positive constant. Then

$$
\ell_{1}\left(\bar{\beta}, \mathcal{W}_{R}\right) \leq \ell_{1}(\bar{\beta}, \Omega)
$$

Proof. Let $v_{p}$ be the positive eigenfunction associated to $\ell_{1}\left(\bar{\beta}, \mathcal{W}_{R}\right)$ and let $\Omega$ be a subset of $\mathcal{W}_{R}$.

Then for every $x \in \partial \Omega$, we can consider $f\left(r_{x}\right)$ as in (4.4) in order to get that the following Robin boundary condition on $\partial \Omega$ holds

$$
\left[F\left(\nabla v_{p}(x)\right)\right]^{p-1} F_{\xi}\left(\nabla v_{p}(x)\right) \cdot \nu+f\left(r_{x}\right) v_{p}(x)^{p-1} F(\nu)=0 .
$$

Having in mind that $\Omega \subset \mathcal{W}_{R}$ and using (5.1), we have that $v_{p}$ solves the following problem

$$
\begin{cases}-\mathcal{Q}_{\mathrm{p}} v_{p}=\ell_{1}\left(\bar{\beta}, \mathcal{W}_{R}\right) v_{p}^{p-1} & \text { in } \Omega \\ {\left[F\left(\nabla v_{p}\right)\right]^{p-1} F_{\xi}\left(\nabla v_{p}\right) \cdot \nu+f\left(r_{x}\right) v_{p}^{p-1} F(\nu)=0} & \text { on } \partial \Omega\end{cases}
$$

Using (5.2) and Lemma 4.2

$$
\begin{aligned}
\ell_{1}\left(\bar{\beta}, \mathcal{W}_{R}\right) & =\frac{\int_{\Omega}\left[F\left(\nabla v_{p}\right)\right]^{p} d x+\int_{\partial \Omega} f\left(r_{x}\right)\left|v_{p}\right|^{p} F(\nu) d \mathcal{H}^{n-1}}{\int_{\Omega}\left|v_{p}\right|^{p} d x} \\
& =\inf _{u \in W^{1, p}(\Omega) \backslash\{0\}} \frac{\int_{\Omega}[F(\nabla u)]^{p} d x+\int_{\partial \Omega} f\left(r_{x}\right)|u|^{p} F(\nu) d \mathcal{H}^{n-1}}{\int_{\Omega}|u|^{p} d x} \\
& \leq \inf _{u \in W^{1, p}(\Omega) \backslash\{0\}} \frac{\int_{\Omega}[F(\nabla u)]^{p} d x+\int_{\partial \Omega} \bar{\beta}|u|^{p} F(\nu) d \mathcal{H}^{n-1}}{\int_{\Omega}|u|^{p} d x} \\
& =\ell_{1}(\bar{\beta}, \Omega)
\end{aligned}
$$

When $\Omega$ contains a Wulff shape we have the following result

Theorem 5.2. Let $\Omega \subset \mathbb{R}^{n}$ be a bounded and convex open set with $C^{1, \alpha}$ boundary, $\left.\alpha \in\right] 0,1[$. Let $\mathcal{W}_{R}$ be a Wulff shape such that $\mathcal{W}_{R} \subset \Omega$, then

$$
\ell_{1}(\bar{\beta}, \Omega) \leq \ell_{1}\left(\bar{\beta}, \mathcal{W}_{R}\right) .
$$

Proof. First of all, we take the positive eigenfunction $v_{p}$ associated to $\ell_{1}\left(\bar{\beta}, \mathcal{W}_{R}\right)$. By Theorem $4.1 v_{p}(x)=\varrho_{p}\left(F^{o}(x)\right)$, and by (4.3) we can extend $\varrho_{p}$ up to $+\infty$ and then $v_{p}$ in $\mathbb{R}^{n}$. Let us consider the super-level set

$$
\mathcal{W}_{+}=\left\{x \in \mathbb{R}^{n}: v_{p}(x)>0\right\} .
$$

By the property of $v_{p} \mathcal{W}_{+}$is a Wulff shape and clearly $\mathcal{W}_{R} \subset \mathcal{W}_{+}$.

Moreover, $v_{p}$ solves the following equation

$$
-\mathcal{Q}_{\mathrm{p}} v_{p}=\ell_{1}\left(\bar{\beta}, \mathcal{W}_{R}\right) v_{p}^{p-1} \text { in } \mathcal{W}_{+} .
$$

To prove the Theorem we consider the set $\tilde{\Omega}=\Omega \cap \mathcal{W}_{+}$. Being $\Omega$ convex and due to the radially decreasing of the eigenfunction, three possible cases can occour. 
Case 1: $\partial \tilde{\Omega}=\partial \Omega$. Then in this case $\mathcal{W}_{R} \subset \Omega \subset \mathcal{W}_{+}$and $\tilde{\Omega}=\Omega$. Then for $x \in \partial \Omega$ we put $r_{x}=F^{o}(x)$ and we can compute

$$
f\left(r_{x}\right)=\frac{\left(-\rho_{p}^{\prime}\left(r_{x}\right)\right)^{p-1}}{\left(\rho_{p}\left(r_{x}\right)\right)^{p-1}} .
$$

Then arguing as in the proof of Theorem 5.1 and recalling that by Lemma 4.2, $f\left(r_{x}\right) \geq \bar{\beta}$, for any $x \in \partial \Omega$ we get

$$
\begin{aligned}
\ell_{1}\left(\bar{\beta}, \mathcal{W}_{R}\right) & =\frac{\int_{\Omega}\left[F\left(\nabla v_{p}\right)\right]^{p} d x+\int_{\partial \Omega} f\left(r_{x}\right)\left|v_{p}\right|^{p} F(\nu) d \sigma}{\int_{\Omega}\left|v_{p}\right|^{p} d x} \\
& =\inf _{u \in W^{1, p}(\Omega) \backslash\{0\}} \frac{\int_{\Omega}[F(\nabla u)]^{p} d x+\int_{\partial \Omega} f\left(r_{x}\right)|u|^{p} F(\nu) d \sigma}{\int_{\Omega}|u|^{p} d x} \\
& \geq \inf _{u \in W^{1, p}(\Omega) \backslash\{0\}} \frac{\int_{\Omega}[F(\nabla u)]^{p} d x+\int_{\partial \Omega} \bar{\beta}|u|^{p} F(\nu) d \sigma}{\int_{\Omega}|u|^{p} d x} \\
& =\ell_{1}(\bar{\beta}, \Omega)
\end{aligned}
$$

and the first case is proved.

Case 2: $\partial \tilde{\Omega} \cap \partial \Omega \neq \emptyset$ and $\partial \tilde{\Omega} \cap \partial \Omega \neq \partial \Omega$. Then $\partial \tilde{\Omega} \cap \mathcal{W}_{+} \neq \emptyset$. Moreover, on $\partial \tilde{\Omega} \cap \partial \Omega$ the eigenfunction $v_{p}$ is positive, while on $\partial \tilde{\Omega} \cap \partial \mathcal{W}_{+}$it is equal to zero. In particular, for every $x \in \partial \tilde{\Omega} \cap \partial \Omega$ we still have that $f\left(r_{x}\right) \geq \bar{\beta}$ as in the Case 1. We define the following test function $\varphi \in W^{1, p}(\Omega)$

$$
\varphi(x)= \begin{cases}v_{p}(x) & \text { in } \tilde{\Omega} \\ 0 & \text { in } \Omega \backslash \tilde{\Omega} .\end{cases}
$$

Then

$$
\begin{aligned}
\ell_{1}\left(\bar{\beta}, \mathcal{W}_{R}\right) & =\frac{\int_{\tilde{\Omega}}\left[F\left(\nabla v_{p}\right)\right]^{p} d x+\int_{\partial \tilde{\Omega} \cap \partial \Omega} f\left(r_{x}\right) v_{p}^{p} F(\nu) d \mathcal{H}^{n-1}}{\int_{\tilde{\Omega}} v_{p}^{p} d x} \\
& =\frac{\int_{\Omega}[F(\nabla \varphi)]^{p} d x+\int_{\partial \tilde{\Omega} \cap \partial \Omega} f\left(r_{x}\right) \varphi^{p} F(\nu) d \mathcal{H}^{n-1}}{\int_{\Omega} v^{p} d x} \\
& \geq \frac{\int_{\Omega}[F(\nabla \varphi)]^{p} d x+\int_{\partial \tilde{\Omega} \cap \partial \Omega} \bar{\beta} \varphi^{p} F(\nu) d \mathcal{H}^{n-1}}{\int_{\Omega} v^{p} d x} \\
& =\frac{\int_{\Omega}[F(\nabla v)]^{p} d x+\int_{\partial \Omega} \bar{\beta} \varphi^{p} F(\nu) d \mathcal{H}^{n-1}}{\int_{\Omega} v^{p} d x}
\end{aligned}
$$




$$
\begin{aligned}
& \geq \inf _{u \in W^{1, p}(\Omega) \backslash\{0\}} \frac{\int_{\Omega}[F(\nabla u)]^{p} d x+\int_{\partial \Omega} \bar{\beta}|u|^{p} F(\nu) d \mathcal{H}^{n-1}}{\int_{\Omega}|u|^{p} d x} \\
& =\ell_{1}(\bar{\beta}, \Omega)
\end{aligned}
$$

and the second case is proved.

Case 3: $\partial \tilde{\Omega} \cap \partial \Omega=\emptyset$. Then $\tilde{\Omega}=\mathcal{W}_{+} \subset \Omega$. Using the monotonicity result in Lemma 4.1 we obtain that $\ell_{1}\left(\bar{\beta}, \mathcal{W}_{R}\right) \geq \ell_{1}\left(\bar{\beta}, \mathcal{W}_{+}\right)$. Denoting with $v_{p}^{(1)}$ the eigenfunction associated to $\ell_{1}\left(\bar{\beta}, \mathcal{W}_{+}\right)$and defining $\tilde{\Omega}^{(1)}=\Omega \cap\left\{v_{p}^{(1)}(x)>0\right\}$ and repeating the division in three possible cases, after a finite number of steps we could be either in Case 1 or in Case 2.

By Theorems 5.1 and 5.2 we get the following monotonicity property for $\ell_{1}$ for constant $\beta$.

Corollary 5.1. Let $\Omega_{1}, \Omega_{2} \subset \mathbb{R}^{n}$ be as in (3.1) and convex. Let $\mathcal{W}_{R}$ be a Wulff shape such that $\Omega_{1} \subset \mathcal{W}_{R} \subset \Omega_{2}$. Then $\ell_{1}\left(\bar{\beta}, \Omega_{2}\right) \leq \ell_{1}\left(\bar{\beta}, \Omega_{1}\right)$.

\section{A representation formula for $\ell_{1}(\beta, \Omega)$}

In this section we prove a level set representation formula for the first eigenvalue $\ell_{1}(\beta, \Omega)$ of the following problem

$$
\begin{cases}-\mathcal{Q}_{\mathrm{p}} v=\ell|v|^{p-2} v & \text { in } \Omega \\ F^{p-1}(\nabla v) F_{\xi}(\nabla v) \cdot \nu+\beta F(\nu)|v|^{p-2} v=0 & \text { on } \partial \Omega .\end{cases}
$$

When $\beta=\bar{\beta}$ is a nonnegative constant a similar result can be found in [5] in the Euclidean case and in [11] for the anisotropic case. Our aim is to extend the known results assuming that $\beta$ is in general a function defined on $\partial \Omega$. In the next we will use the following notation. Let $\tilde{u}_{p}$ be the first positive eigenfunction such that $\max \tilde{u}_{p}=1$. Then, for $t \in[0,1]$,

$$
\begin{aligned}
& U_{t}=\left\{x \in \Omega: \tilde{u}_{p}>t\right\}, \\
& S_{t}=\left\{x \in \Omega: \tilde{u}_{p}=t\right\}, \\
& \Gamma_{t}=\left\{x \in \partial \Omega: \tilde{u}_{p}>t\right\} .
\end{aligned}
$$

Theorem 6.1. Let $\Omega \subset \mathbb{R}^{n}$ be a bounded open set with $C^{1, \alpha}$ boundary and let $\left.\alpha \in\right] 0,1[$. Let $\beta$ be a function belonging to $L^{1}(\partial \Omega), \beta \geq 0$ and such that (3.3) holds. Let $\tilde{u}_{p} \in C^{1, \alpha}(\Omega) \cap L^{\infty}(\Omega)$ be a positive minimizer of (3.5) with $\left\|\tilde{u}_{p}\right\|_{\infty}=1$. Then for a.e. $\left.t \in\right] 0,1[$ the following representation formula holds

$$
\ell_{1}(\beta, \Omega)=\mathcal{F}_{\Omega}\left(U_{t}, \frac{\left[F\left(\nabla \tilde{u}_{p}\right)\right]^{p-1}}{\tilde{u}_{p}^{p-1}}\right),
$$

where $\mathcal{F}_{\Omega}$ is defined as

$$
\mathcal{F}_{\Omega}\left(U_{t}, \varphi\right)=\frac{1}{\left|U_{t}\right|}\left(-(p-1) \int_{U_{t}} \varphi^{p^{\prime}} d x+\int_{S_{t}} \varphi F(\nu) d \sigma+\int_{\Gamma_{t}} \beta F(\nu) d \sigma\right) .
$$

Proof. Let $0<\varepsilon<t<1$ and we define

$$
\psi_{\varepsilon}= \begin{cases}0 & \text { if } \tilde{u}_{p} \leq t \\ \frac{\tilde{u}_{p}}{\varepsilon} \frac{1}{\tilde{u}_{p}^{p-1}} & \text { if } t<\tilde{u}_{p}<t+\varepsilon \\ \frac{1}{\tilde{u}_{p}^{p-1}} & \text { if } \tilde{u}_{p} \geq t+\varepsilon .\end{cases}
$$


The functions $\psi_{\varepsilon}$ are in $W^{1, p}(\Omega)$ and increasingly converge to $\tilde{u}_{p}^{-(p-1)} \chi_{U_{t}}$ as $\varepsilon \rightarrow 0$.

Moreover, we can obtain that

$$
\nabla \psi_{\varepsilon}= \begin{cases}0 & \text { if } \tilde{u}_{p}<t \\ \frac{1}{\varepsilon}\left((p-1) \frac{t}{\tilde{u}_{p}}+2-p\right) \frac{\nabla \tilde{u}_{p}}{\tilde{u}_{p}^{p-1}} & \text { if } t<\tilde{u}_{p}<t+\varepsilon \\ -(p-1) \frac{\nabla \tilde{u}_{p}}{\tilde{u}_{p}^{p}} & \text { if } \tilde{u}_{p}>t+\varepsilon .\end{cases}
$$

Then choosing $\psi_{\varepsilon}$ as test function in (3.4), we get that the first integral is

$$
\begin{aligned}
& -(p-1) \int_{U_{t+\varepsilon}} \frac{\left[F\left(\nabla \tilde{u}_{p}\right)\right]^{p}}{\tilde{u}_{p}^{p}} d x+\frac{1}{\varepsilon} \int_{U_{t} \backslash U_{t+\varepsilon}} \frac{\left[F\left(\nabla \tilde{u}_{p}\right)\right]^{p}}{\tilde{u}_{p}^{p-1}}\left((p-1) \frac{t}{\tilde{u}_{p}}+2-p\right) d x= \\
& =-(p-1) \int_{U_{t+\varepsilon}} \frac{\left[F\left(\nabla \tilde{u}_{p}\right)\right]^{p}}{\tilde{u}_{p}^{p}} d x+\frac{1}{\varepsilon} \int_{t}^{t+\varepsilon}\left((p-1) \frac{t}{\tau}+2-p\right) \int_{S_{\tau}} \frac{\left[F\left(\nabla \tilde{u}_{p}\right)\right]^{p-1}}{\tilde{u}_{p}^{p-1}} F(\nu) d \mathcal{H}^{n-1},
\end{aligned}
$$

where last equality follows by the coarea formula. Then, reasoning as in [5] and [1] we get that

$$
\int_{\Omega}\left[F\left(\nabla \tilde{u}_{p}\right)\right]^{p-1} F_{\xi}\left(\nabla \tilde{u}_{p}\right) \cdot \nabla \psi_{\varepsilon} d x \stackrel{\varepsilon \rightarrow 0}{\longrightarrow}-(p-1) \int_{U_{t}} \frac{\left[F\left(\nabla \tilde{u}_{p}\right)\right]^{p}}{\tilde{u}_{p}^{p}} d x+\int_{S_{t}} \frac{\left[F\left(\nabla \tilde{u}_{p}\right)\right]^{p-1}}{\tilde{u}_{p}^{p-1}} F(\nu) d \mathcal{H}^{n-1} .
$$

As regards the other two integrals in (3.4), we have

$$
\begin{array}{r}
\int_{\partial \Omega} \beta \tilde{u}_{p}^{p-1} \psi_{\varepsilon} F(\nu) d \mathcal{H}^{n-1}=\int_{\Gamma_{t+\varepsilon}} \beta F(\nu) d \mathcal{H}^{n-1}+\int_{\Gamma_{t} \backslash \Gamma_{t+\varepsilon}} \beta \frac{\tilde{u}_{p}-t}{\varepsilon} F(\nu) d \mathcal{H}^{n-1} \stackrel{\varepsilon \rightarrow 0}{\longrightarrow}, \\
\stackrel{\varepsilon \rightarrow 0}{\longrightarrow} \int_{\Gamma_{t}} \beta F(\nu) d \mathcal{H}^{n-1},
\end{array}
$$

by dominated convergence theorem and by monotone convergence theorem and the definition of $\psi_{\varepsilon}$

$$
\ell_{1}(\beta, \Omega) \int_{\Omega} \tilde{u}_{p}^{p-1} \psi_{\varepsilon} d x \stackrel{\varepsilon \rightarrow 0}{\longrightarrow} \ell_{1}(\beta, \Omega)\left|U_{t}\right|
$$

Summing the three limits, we get (6.2).

When we consider a generic test function we have

Theorem 6.2. Let $\Omega \subset \mathbb{R}^{n}$ be a bounded open set with $C^{1, \alpha}$ boundary and let $\left.\alpha \in\right] 0,1[$. Let $\varphi$ be a nonnegative function in $\Omega$ such that $\varphi \in L^{p^{\prime}}(\Omega)$, where $p^{\prime}=\frac{p}{p-1}$. If $\varphi \not \equiv\left[F\left(\nabla \tilde{u}_{p}\right)\right]^{p-1} / \tilde{u}_{p}^{p-1}$, where $\tilde{u}_{p}$ is the eigenfunction given in Theorem [6.1, and $\mathcal{F}_{\Omega}$ is the functional defined in (6.3), then there exists a set $S \subset] 0,1[$ with positive measure such that for every $t \in S$ it holds that

$$
\ell_{1}(\beta, \Omega)>\mathcal{F}_{\Omega}\left(U_{t}, \varphi\right) .
$$

The proof is similar to that obtained in [5] and [11], and we only sketch it here. It can be divided in two main steps. First, we claim that, if

$$
w(x):=\varphi-\frac{\left[F\left(\nabla \tilde{u}_{p}\right)\right]^{p-1}}{\tilde{u}_{p}^{p-1}}, \quad I(t):=\int_{U_{t}} w \frac{F\left(\nabla \tilde{u}_{p}\right)}{\tilde{u}_{p}} d x
$$

then the function $I:] 0,1[\rightarrow \mathbb{R}$ is locally absolutely continuous and

$$
\mathcal{F}_{\Omega}\left(U_{t}, \varphi\right) \leq \ell_{1}(\beta, \Omega)-\frac{1}{\left|U_{t}\right| t^{p-1}}\left(\frac{d}{d t}\left(t^{p} I(t)\right)\right)
$$


for almost every $t \in] 0,1\left[\right.$. Second, we show that the derivative $\frac{d}{d t}\left(t^{p} I(t)\right)$ is positive in a subset of $] 0,1[$ with nonzero measure.

In order to prove (6.5), using the representation formula (6.2) we obtain that, for a.e. $t \in] 0,1[$,

$$
\begin{aligned}
\mathcal{F}_{\Omega}\left(U_{t}, \varphi\right) & =\ell_{1}(\beta, \Omega)+\frac{1}{\left|U_{t}\right|}\left(\int_{S_{t}} w F(\nu) d \mathcal{H}^{n-1}-(p-1) \int_{U_{t}}\left(\varphi^{p^{\prime}}-\frac{\left[F\left(\nabla \tilde{u}_{p}\right)\right]^{p}}{\tilde{u}_{p}^{p}}\right) d x\right) \\
& \leq \ell_{1}(\beta, \Omega)+\frac{1}{\left|U_{t}\right|}\left(\int_{S_{t}} w F(\nu) d \mathcal{H}^{n-1}-p \int_{U_{t}} w \frac{F\left(\nabla \tilde{u}_{p}\right)}{\tilde{u}_{p}} d x\right) \\
& =\ell_{1}(\beta, \Omega)+\frac{1}{\left|U_{t}\right|}\left(\int_{S_{t}} w F(\nu) d \mathcal{H}^{n-1}-p I(t)\right)
\end{aligned}
$$

where the inequality in (6.6) follows from the inequality $\varphi^{p^{\prime}} \geq v^{p^{\prime}}+p^{\prime} v^{p^{\prime}-1}(\varphi-v)$, with $\varphi, v \geq 0$. Proceeding as in [11] and using the coarea formula we, obtain for a.e. $t \in] 0,1[$

$$
-\frac{d}{d t}\left(t^{p} I(t)\right)=t^{p-1}\left(\int_{S_{t}} w F(\nu) d \mathcal{H}^{n-1}-p I(t)\right) .
$$

Substituting (6.7) in (6.6) we obtain (6.5). We can conclude the proof, arguing by contradiction exactly as in [5, Theorem 3.2], indeed is possible to see that the function $t^{p} I(t)$ has positive derivative in a set of positive measure. This fact with (6.5) give us the inequality (6.4).

\subsection{Applications}

In this section we use the representation formula given in Theorem 6.1 in order to get some estimates for $\ell_{1}(\beta, \Omega)$.

\subsubsection{A Faber-Krahn type inequality}

Let $\Omega \subset \mathbb{R}^{n}$ be a bounded open set with $C^{1, \alpha}$ boundary, $\left.\alpha \in\right] 0,1\left[\right.$ and let $\mathcal{W}_{R}$ be the Wulff shape centered at the origin with radius $R$ such that $|\Omega|=\left|\mathcal{W}_{R}\right|$.

Let $\bar{\beta}$ be a positive constant and let us consider the following Robin eigenvalue problem in $\mathcal{W}_{R}$ for $\mathcal{Q}_{\mathrm{p}}$

$$
\begin{cases}-\mathcal{Q}_{\mathrm{p}} v=\ell_{1}\left(\bar{\beta}, \mathcal{W}_{R}\right)|v|^{p-2} v & \text { in } \mathcal{W}_{R}, \\ F^{p-1}(\nabla v) F_{\xi}(\nabla v) \cdot \nu+\bar{\beta} F(\nu)|v|^{p-2} v=0 & \text { on } \partial \mathcal{W}_{R}\end{cases}
$$

Let $w(t), t \in[0,+\infty[$, be a non negative continuous function such that

$$
w(t) \geq C(R) t,
$$

where $C(R)=\ell_{1}\left(\bar{\beta}, \mathcal{W}_{R}\right)+(p-1) \bar{\beta}^{\frac{p}{p-1}}$ is the constant appearing in (4.7).

Let us consider the following Robin eigenvalue problem

$$
\begin{cases}-\mathcal{Q}_{\mathrm{p}} u=\ell_{1}(\beta, \Omega)|u|^{p-2} u & \text { in } \Omega, \\ F^{p-1}(\nabla u) F_{\xi}(\nabla u) \cdot \nu+\beta(x) F(\nu)|u|^{p-2} u=0 & \text { on } \partial \Omega,\end{cases}
$$

where

$$
\beta(x)=w\left(F^{o}(x)\right), \quad x \in \partial \Omega .
$$

As a consequence of the representation formula (6.1) for $\ell_{1}(\beta, \Omega)$ we get the following FaberKrahn inequality. 
Theorem 6.3. Let $\Omega \subset \mathbb{R}^{n}$ be a bounded open set with $C^{1, \alpha}$ boundary, let $\left.\alpha \in\right] 0,1\left[\right.$ and let $\mathcal{W}_{R}$ be the Wulff shape such that $|\Omega|=\left|\mathcal{W}_{R}\right|$. Let $w(t), t \in[0,+\infty[$, be a non negative continuous function which verifies (6.9) and let $\beta(x)$ be the function defined in (6.11). Then,

$$
\ell_{1}\left(\bar{\beta}, \mathcal{W}_{R}\right) \leq \ell_{1}(\beta, \Omega) .
$$

Proof. We construct a suitable test function in $\Omega$ for (6.4). Let $v_{p}$ be a positive eigenfunction of the radial problem (6.8) in $B_{R}$. By Theorem 4.1, $v_{p}$ is a function depending only by $F^{o}(x)$, $v_{p}=\rho_{p}\left(F^{o}(x)\right)$, and then we can argue as in Section 4 defining the function

$$
f\left(r_{x}\right)=\varphi_{\star}(x)=\frac{\left[F\left(\nabla v_{p}(x)\right)\right]^{p-1} F_{\xi}\left(D v_{p}(x)\right) \cdot \nu}{v_{p}(x)^{p-1} F(\nu)}=\frac{\left(-\rho_{p}^{\prime}\left(r_{x}\right)\right)^{p-1}}{\left(\rho_{p}\left(r_{x}\right)\right)^{p-1}},
$$

where $r_{x}=F^{o}(x) \in[0, R]$

Denoted by $\mathcal{W}_{s}=\left\{x \in \mathcal{W}_{R}: v_{p}(x)>s\right\}, 0<s<R$, clearly $\mathcal{W}_{s}$ is a Wulff shape centered at the origin and by Theorem (6.1) we get

$$
\ell_{1}\left(\beta(R), \mathcal{W}_{R}\right)=F_{\mathcal{W}_{R}}\left(\mathcal{W}_{s}, \varphi_{\star}\right)=\frac{1}{\left|\mathcal{W}_{s}\right|}\left(-(p-1) \int_{\mathcal{W}_{s}} \varphi_{\star}^{p^{\prime}} d x+\int_{\partial \mathcal{W}_{s}} \varphi_{\star} F(\nu) d \mathcal{H}^{n-1}\right)
$$

Let $\tilde{u}_{p}$ be the first eigenfunction of (6.10) in $\Omega$ such that $\left\|\tilde{u}_{p}\right\|_{\infty}=1$. For $x \in \Omega$ we set $\tilde{u}_{p}(x)=t, 0<t<1$. Then we consider the Wulff shape $\mathcal{W}_{r(t)}$, centered at the origin, where $r(t)$ is the positive number such that $\left|U_{t}\right|=\left|\mathcal{W}_{r(t)}\right|$. Then, we define the following test function

$$
\varphi(x):=f(r(t))=f\left(F^{o}(x)\right) .
$$

We stress that clearly $r(t)<R$. Our aim is to compare $\mathcal{F}_{\Omega}\left(U_{t}, \varphi\right)$ with $\mathcal{F}_{\mathcal{W}_{R}}\left(\mathcal{W}_{r(t)}, \varphi_{\star}\right)$. Then by (6.13) with $s=r(t)$ we have to show that

$$
\begin{aligned}
\mathcal{F}_{\Omega}\left(U_{t}, \varphi\right) & \geq \frac{1}{\left|\mathcal{W}_{r(t)}\right|}\left(-(p-1) \int_{\mathcal{W}_{r(t)}} \varphi_{\star}^{p^{\prime}} d x+\int_{\partial \mathcal{W}_{r(t)}} \varphi_{\star} F(\nu) d \mathcal{H}^{n-1}\right) \\
& =\mathcal{F}_{\mathcal{W}_{R}}\left(\mathcal{W}_{r(t)}, \varphi_{\star}\right) .
\end{aligned}
$$

We first observe that by [26, Section 1.2.3], being $\left|U_{t}\right|=\left|\mathcal{W}_{r(t)}\right|$ for all $\left.t \in\right] 0,1[$

$$
\int_{U_{t}} \varphi^{p^{\prime}} d x=\int_{\mathcal{W}_{r(t)}} \varphi_{\star}^{p^{\prime}} d x
$$

Moreover, from the weighted isoperimetric inequality quoted in Remark 4.2. Theorem 4.3 and the assumption (6.11) on $\beta$ we get

$$
\begin{aligned}
& \int_{\partial \mathcal{W}_{r(t)}} \varphi_{\star} F(\nu) d \sigma=\int_{\partial \mathcal{W}_{r(t)}} f(r(t)) F(\nu) d \mathcal{H}^{n-1} \leq \int_{\partial U_{t}} f\left(F^{o}(x)\right) F(\nu) d \mathcal{H}^{n-1} \leq \\
& \leq \int_{S_{t}} f\left(F^{o}(x)\right) F(\nu) d \mathcal{H}^{n-1}+\int_{\Gamma_{t}} f\left(F^{o}(x)\right) F(\nu) d \mathcal{H}^{n-1} \\
& =\int_{S_{t}} \varphi F(\nu) d \mathcal{H}^{n-1}+\int_{\Gamma_{t}} f\left(F^{o}(x)\right) F(\nu) d \mathcal{H}^{n-1} \\
& \leq \int_{S_{t}} \varphi F(\nu) d \mathcal{H}^{n-1}+C(R) \int_{\Gamma_{t}} F^{o}(x) F(\nu) d \mathcal{H}^{n-1} \\
& \leq \int_{S_{t}} \varphi F(\nu) d \mathcal{H}^{n-1}+\int_{\Gamma_{t}} w\left(F^{o}(x)\right) F(\nu) d \mathcal{H}^{n-1}
\end{aligned}
$$

and this concludes the proof.

Remark 6.1. When $\beta=\bar{\beta}$ is a nonnegative constant (6.12) is proved in [11] in the anisotropic case and in [5, 8] in the Euclidean case. 


\subsubsection{A Cheeger type inequality for $\ell_{1}(\beta, \Omega)$}

In this part we introduce the anisotropic weighted Cheeger constant and, using the representation formula we prove an anisotropic weighted Cheeger inequality for $\ell_{1}(\beta, \Omega)$. Following [7] we give

Definition 6.1. Let $g: \bar{\Omega} \rightarrow] 0, \infty[$ be a continuous function the weighted anisotropic Cheeger constant is defined as follows

$$
h_{g}(\Omega)=\inf _{U \subset \Omega} \frac{\int_{\partial U} g F(\nu) d \mathcal{H}^{n-1}}{|U|}=\inf _{U \subset \Omega} \frac{P_{F}(g, U)}{|U|} .
$$

We observe that when $g(x)=c$ is a constant then

$$
h_{g}(\Omega)=c \inf _{U \subset \Omega} \frac{P_{F}(U)}{|U|}=\operatorname{ch}(\Omega),
$$

where $h(\Omega)$ is the anisotropic Cheeger constant defined in (2.7) . In [7] it is proved that actually $h_{g}(\Omega)$ is a minimum that is there exists a set $C \subset \Omega$ such that

$$
h_{g}(\Omega)=\frac{P_{F}(g, C)}{|C|}
$$

and we refer to $C$ as a weighted Cheeger set.

We observe that for suitable weight $g$ the constant $h_{g}(\Omega)$ verifies an anisotropic isoperimetric inequality

Theorem 6.4. Let $g(x)=w\left(F^{o}(x)\right)=w(r), r \geq 0$ with $w$ a non negative and nondecreasing function such that

$$
w\left(r^{\frac{1}{n}}\right) r^{1-\frac{1}{n}}, \quad 0 \leq r \leq R^{n},
$$

is convex with respect to $r$. Then

$$
h_{g}(\Omega) \geq h_{g}\left(\mathcal{W}_{R}\right)=\frac{n w(R)}{R},
$$

where $\mathcal{W}$ is a Wulff shape with the same measure as $\Omega$.

Proof. The proof follows immediately from Remark 4.2 .

When $\beta=\bar{\beta}$ is a nonnegative constant and $p=2$ in $[22$ the following Cheeger inequality is proved in the Robin eigenvalue case

$$
\ell_{1}(\bar{\beta}, \Omega) \geq \begin{cases}h(\Omega) \bar{\beta}-\bar{\beta}^{2} & \text { always } \\ \frac{1}{4}[h(\Omega)]^{2} & \text { if } \bar{\beta} \geq \frac{1}{2} h(\Omega)\end{cases}
$$

In the next result we extend (6.14) to the anisotropic case for any $1<p<\infty$ considering $\beta$ not in general constant.

Theorem 6.5. Let us consider problem (6.1) with $\beta \in C(\bar{\Omega})$ such that $\beta \geq 0$. Then the following weighted anisotropic Cheeger inequality holds

$$
\ell_{1}(\beta, \Omega) \geq h_{\beta}(\Omega)-(p-1)\left\|\beta^{p^{\prime}}\right\|_{L^{\infty}(\bar{\Omega})},
$$

where $p^{\prime}=\frac{p}{p-1}$. 
Proof. Using $\beta$ as test function in (6.4) we obtain

$$
\begin{aligned}
\ell_{1}(\beta, \Omega) \geq & \mathcal{F}\left(U_{t}, \beta\right)=\frac{1}{\left|U_{t}\right|}\left(-(p-1) \int_{U_{t}} \beta^{p^{\prime}} d x+\int_{S_{t}} \beta F(\nu) d \mathcal{H}^{n-1}+\int_{\Gamma_{t}} \beta F(\nu) d \mathcal{H}^{n-1}\right) \\
& =\frac{1}{\left|U_{t}\right|}\left(-(p-1) \int_{U_{t}} \beta^{p^{\prime}} d x+\int_{\partial U_{t}} \beta F(\nu) d \mathcal{H}^{n-1}\right) \geq-(p-1)\left\|\beta^{p^{\prime}}\right\|_{\infty}+h_{\beta}(\Omega) .
\end{aligned}
$$

Remark 6.2. We observe that the previous result continues to hold if we take $\beta \in C(\partial \Omega)$. Indeed in this case from a classical result, see for instance [18, Theorem 4.I], we know that the function $\beta$ is the trace of a nonnegative function $\beta_{\Omega} \in C(\bar{\Omega})$. Then inequality (6.15) holds with $\beta=\beta_{\Omega}$.

We emphasize the inequality (6.15) in the particular case of $\beta=\bar{\beta}$ is a nonnegative constant.

Corollary 6.1. The first eigenvalue $\ell_{1}(\bar{\beta}, \Omega)$ of (6.1) on a fixed bounded open set $\Omega \subset \mathbb{R}^{n}$ with Lipschitz boundary satisfies

$$
\ell_{1}(\bar{\beta}, \Omega) \geq \begin{cases}h(\Omega) \bar{\beta}-(p-1) \bar{\beta}^{\frac{p}{p-1}} & \text { always } \\ \frac{1}{p^{p}}[h(\Omega)]^{p} & \text { if } \bar{\beta} \geq \frac{1}{p^{p-1}}[h(\Omega)]^{p-1}\end{cases}
$$

Proof. From the Theorem 6.5 we obtain, using the constant function $\bar{\beta}$ as test, we obtain the first part of the inequality. For the second part, is suitable using as test function in the functional $\mathcal{F}_{\Omega}\left(U_{t}, \cdot\right)$, the constant $\frac{1}{p^{p-1}}\left[h_{F}(\Omega)\right]^{p-1}$ under the assumption that the constant $\bar{\beta} \geq \frac{1}{p^{p-1}}\left[h_{F}(\Omega)\right]^{p-1}$.

Remark 6.3. From the anisotropic Cheeger inequality for constant $\bar{\beta}$ we obtain immediately a lower bound for $\ell_{1}(\bar{\beta}, \Omega)$ in terms of the anisotropic inradius of $\Omega$ different from (3.8) by using (2.8).

Remark 6.4. By (ii) Theorem 3.1 and Corollary 6.1 we obtain for $\bar{\beta} \geq \frac{1}{p^{p-1}}[h(\Omega)]^{p-1}$ the anisotropic Cheeger inequality (6.16) for the first Dirichlet eigenvalue of $\mathcal{Q}_{\mathrm{p}}$

$$
\lambda_{D}(\Omega) \geq \ell_{1}(\bar{\beta}, \Omega) \geq \frac{1}{p^{p}}[h(\Omega)]^{p} .
$$

\section{References}

[1] A. Alvino, V. Ferone, P. - L. Lions, G. Trombetti, Convex symmetrization and applications, Ann. Inst. H. Poincaré Anal. Non Linéaire, 14. 2. (1997): 275-293.

[2] M. Belloni, V. Ferone, and B. Kawohl. Isoperimetric inequalities, Wulff shape and related questions for strongly nonlinear elliptic operators. Zeitschrift fur Angewandte Mathematik und Physik (ZAMP), 54. 5. (2003): 771-783.

[3] M. F. Betta, F. Brock, A. Mercaldo, M. R. Posteraro, Weighted isoperimetric inequalities on $\mathbb{R}^{n}$ and applications to rearrangements. Math. Nachr. 281. 4. (2008): 466-498.

[4] L. Brasco, G. Franzina, An anisotropic eigenvalue problem of Stekloff type and weighted Wulff inequalities. NoDEA. Nonlinear Differential Equations and Appl. 20. 6. (2013): 17951830

[5] D. Bucur, D. Daners, An alternative approach to the Faber-Krahn inequality for Robin problems. Calc. Var. 37. (2010): 75-86 
[6] H. Busemann. The isoperimetric problem for Minkowski area. Amer. J. Math., 71. (1949): 743-762.

[7] V. Caselles, G. Facciolo, E. Meinhardt, Anisotropic Cheeger Sets and Applications. SIAM J. Imaging Sci. 2. 4. (2009): 1211-1254.

[8] Q.-y. Dai, Y.-x. Fu, Faber-Krahn inequality for Robin problems involving p-Laplacian. Acta Mathematica Applicatae Sinica, English Series. 27. (2011): 13-28

[9] D.Daners, A Faber-Krahn inequality for Robin problems in any space dimension. Mathematische Annalen. 335. (2006): 767-785.

[10] F. Della Pietra, G. di Blasio, N. Gavitone, Sharp estimates on the first Dirichlet eigenvalue of nonlinear elliptic operators via maximum principle, arXiv:1710.03140.

[11] F. Della Pietra, N. Gavitone, Faber-Krahn Inequality for Anisotropic Eigenvalue Problems with Robin Boundary Conditions. Potential Analysis. 41. (2014): 1147-1166

[12] F. Della Pietra, N. Gavitone, S. Guarino lo Bianco, On functionals involving the tor- sional rigidity related to some classes of nonlinear operators. arXiv:1705.03330.

[13] F. Della Pietra, N. Gavitone, H. Kovařik, Optimizing the First Eigenvalue of some Quasilinear Operators with respect to Boundary Conditions. ESAIM: COCV. 23. (2017): 1381-1395.

[14] F. Della Pietra, N. Gavitone, G. Piscitelli, On the second Dirichlet eigenvalue of some nonlinear anisotropic elliptic operators. arXiv:1704.00508.

[15] E. N. Dancer, D. Daners, Domain perturbation for elliptic equations subject to Robin boundary conditions. J. Differential Equations. 138. (1997): 86-132.

[16] E. DiBenedetto, $C^{1+\alpha}$ local regularity of weak solutions of degenerate elliptic equations. Nonlinear Anal. 7. 8. (1983): 827-850.

[17] I. Fonseca and S. Müller. A uniqueness proof for the Wulff theorem. Proc. Roy. Soc. Edinburgh Sect. A. 119. 1-2. (1991): 125-136.

[18] E. Gagliardo, Caratterizzazioni delle tracce sulla frontiera relative ad alcune classi di funzioni in $n$ variabili. Rend. Sem. Mat. Univ. Padova. 27. (1957) 284-305

[19] D. Gilbarg, N. S. Trudinger, Elliptic partial differential equations of second order, 2nd edn. Springer-Verlag (1983)

[20] T. Giorgi, R. G. Smits, Monotonicity results for the principal eigenvalue of the generalized Robin Problem. Illinois Journal of Mathematics. 49. 4 (2005): 1133-1143

[21] B. Kawohl, M. Novaga, The p-Laplace eigenvalue problem as $p \rightarrow 1$ and Cheeger sets in a Finsler metric. J. Convex Anal. 15. 3. (2008): 623-634

[22] J. B. Kennedy, On the isoperimetric problem for the Laplacian with Robin and Wentzell boundary conditions. University of Sydney. School of Mathematics and Statistics. Ph.D. Thesis (2010)

[23] J. R. Kuttler, A Note on a Paper of Sperb. ZAMP. 24. 3. (1973): 431-434

[24] O. A. Ladyzhenskaya, N. N. Ural'tseva Linear and quasilinear elliptic equations. Translated from the Russian by Scripta Technica Inc. Translation editor: Leon Ehrenpreis. Academic Press, New York. (1968)

[25] P. Lindqvist, On a Nonlinear Eigenvalue Problem. Rocky Mountain Journal of Mathematics. 23. 1. (1993) 
[26] V. G. Maz'ja, Sobolev Spaces. Springer Verlag, Berlin (1985)

[27] P. Tolksdorf, Regularity for a more general class of quasilinear elliptic equations. J. Diff. Equat. 54. 1. (1984): 126-150

[28] N. S. Trudinger, On Harnack type inequalities and their application to quasilinear elliptic equations. Comm. Pure Appl. Math. 20. (1967): 721-747 